\begin{tabular}{|c|c|}
\hline Title & The stability condition for the FDTD of the optical diffusion equations \\
\hline Author(s) & Toba, Hidemitsu; Odate, Satoru; Otaki, Katsura; Nishimura, Goro \\
\hline Citation & $\begin{array}{l}\text { The Optical Society of Japan (日本光学会) ，27(1), 81-89 } \\
\text { https://doi.org/10.1007/s10043-019-00567-7 }\end{array}$ \\
\hline Issue Date & $2020-02$ \\
\hline Doc URL & http:/hdl. handle.net/2115/80344 \\
\hline Rights & $\begin{array}{l}\text { This is a post-peer-review, pre copyedit version of an article published in Optical Review. The final authenticated } \\
\text { version is available online at: http://dx.doi.org/10.1007/s10043-019-00567-7 }\end{array}$ \\
\hline Type & article (author version) \\
\hline File Information & Draft20191011.pdf \\
\hline
\end{tabular}

Instructions for use 


\title{
The stability condition for the FDTD of the optical diffusion equations
}

\author{
Hidemitsu Toba · Satoru Odate · Katsura \\ Otaki · Goro Nishimura
}

Received: date / Accepted: date

\begin{abstract}
The Finite-difference time-domain (FDTD) method, which is often used to solve Maxwell's equations, has been proposed to solve the optical diffusion equations to simulate light propagation in turbid media such as biological tissue. Finite difference methods can be numerically unstable and calculation can diverge unless a stability condition is satisfied. Until now, the stability condition for FDTD of the optical diffusion equations has not been derived. In this paper, we derive a stability condition for FDTD of the optical diffusion equations by using the von-Neumann stability analysis and verify the condition numerically.
\end{abstract}

Keywords optical diffusion equations $\cdot$ FDTD $\cdot$ stability analysis

H. Toba

Nikon corporation, 471, Nagaodai-cho, Sakae-ku, Yokohama-city, Kanagawa, Japan

Tel.: +81-45-853-8445

Fax: +81-45-853-8519

E-mail: Hidemitsu.Toba@nikon.com

S. Odate

Nikon corporation, 471, Nagaodai-cho, Sakae-ku,Yokohama-city, Kanagawa, Japan

Tel.: +81-45-853-8445

Fax: +81-45-853-8519

K. Otaki

Nikon corporation, 471, Nagaodai-cho, Sakae-ku, Yokohama-city, Kanagawa, Japan

Tel.: +81-45-853-8445

Fax: +81-45-853-8519

G. Nishimura

Research Institute for Electronic Science, Hokkaido University N20W10, Kita-ku, Sapporo, Hokkaido, Japan 


\section{Introduction}

Investigation of biological tissues with light is an active area of research and development, and one key technique is diffused optical tomography. Visible and near-infrared light is strongly scattered in turbid media such as biological tissues. Therefore it is often almost impossible to apply a conventional light propagation theory such as the Fresnel-Kirchhoff diffraction theory because of the strong multiple scattering. The Radiative Transport Equation (RTE) is known as a rigorous light propagation theory in such media. However, because it is computationally hard to solve, the diffusion approximation is often applied to the RTE. This approximation is not satisfied in some cases, e.g., near tissue surfaces. To overcome this issue, several higher order approximations to the RTE have been developed[1-6]. While these developments improve the accuracy of the calculation, the diffusion approximation is still useful and widely employed for reducing the calculation time [7]

Several techniques have been developed to solve diffusion equation(s) numerically including the Finite Element Method (FEM) [8], the Monte Carlo Method(MC) [7] and the finite-difference time-domain method or the FDTD $[9,10]$.

FDTD is a very simple algorithm. It is easy to parallelize FDTD and estimate the required memory as the data size increases. FDTD for the Maxwell's equations, originally proposed by Yee [11], is one of the most popular applications of FDTD. Yee's scheme can solve electromagnetic problems in wavelength scale, and it has become an indispensable tool with many commercial software products now available to engineers in optics and photonics. It may be possible to use FDTD for the diffusion equations as widely as it is used for Maxwell's equations.

The finite difference methods solve the differential equations by approximating them as difference equations. Finite difference calculations are executed numerically and inevitably include round-off errors. Without proper conditioning, the finite difference calculation can cause the numerical error to grow exponentially, resulting in a large deviation from the exact answer. The general stability condition for solving finite difference equations numerically has been studied for decades. The von Neumann stability analysis is the most popular procedure for determining the stability of finite difference schemes [12]. Until now, the stability condition for the FDTD method applied to the diffusion equations has not yet been derived.

We review FDTD formulated for the diffusion equations in section 2 . We derive the stability condition of FDTD for the diffusion equations in section 3 . And then we verify the stability condition numerically in section 4 .

\section{FDTD formulated for diffusion equations}

The time-dependent partial differential optical diffusion equations in Cartesian coordinates are expressed as[10] 


$$
\begin{aligned}
& \frac{1}{v} \frac{\partial \boldsymbol{J}(\boldsymbol{r}, t)}{\partial t}+\frac{1}{3} \nabla \phi(\boldsymbol{r}, t)+\mu_{t r} \boldsymbol{J}(\boldsymbol{r}, t)=\int_{(4 \pi)} \mathrm{d}^{2} \hat{\boldsymbol{s}} \hat{\boldsymbol{s}} \varepsilon_{r i}(\boldsymbol{r}, t) \\
& \frac{1}{v} \frac{\partial \phi(\boldsymbol{r}, t)}{\partial t}+\nabla \cdot \boldsymbol{J}(\boldsymbol{r}, t)+\mu_{a} \phi(\boldsymbol{r}, t)=\int_{(4 \pi)} \mathrm{d}^{2} \hat{\boldsymbol{s}} \varepsilon_{r i}(\boldsymbol{r}, t) \\
& \varepsilon_{r i}(\boldsymbol{r}, \hat{\boldsymbol{s}}, t)=\frac{\mu_{a}+\mu_{s}}{4 \pi} \int_{(4 \pi)} \mathrm{d}^{2} \hat{\boldsymbol{s}}^{\prime} p\left(\hat{\boldsymbol{s}}, \hat{\boldsymbol{s}}^{\prime}\right) I_{r i}\left(\boldsymbol{r}, \hat{\boldsymbol{s}}^{\prime}, t\right) \\
& \mu_{t r}=\mu_{a}+\mu_{s}^{\prime} \\
& \mu_{s}^{\prime}=(1-g) \mu_{s}
\end{aligned}
$$

where $\phi(\boldsymbol{r}, t), \boldsymbol{J}(\boldsymbol{r}, t)$ and $I_{r i}(\boldsymbol{r}, \hat{\boldsymbol{s}}, t)$ are the photon fluence rate of light, the photon flux and the reduced incident intensity at position $\boldsymbol{r}$ at time $t$ with velocity in direction $\hat{\boldsymbol{s}}$ respectively, and $\mu_{a}, \mu_{s}$ and $g$ are the absorption coefficient, scattering coefficient and the anisotropy factor. $v=c / n_{m}$ is the speed of light in media with the refractive index $n_{m} \cdot p\left(\hat{\boldsymbol{s}}, \hat{\boldsymbol{s}}^{\prime}\right)$ is called the scattering phase function. In the right hand side of the three equations, integration is over the whole unit sphere.

If the time derivative of the photon flux is negligible, $v^{-1} \partial \boldsymbol{J} / \partial t \sim 0$, one can obtain the conventional diffusion equation [7]

$$
\left(\frac{1}{v} \frac{\partial}{\partial t}-\nabla \cdot\left(\frac{1}{3 \mu_{t r}} \nabla\right)+\mu_{a}\right) \phi(\boldsymbol{r}, t)=\int_{(4 \pi)} \mathrm{d}^{2} \hat{\boldsymbol{s}} \varepsilon_{r i}(\boldsymbol{r}, t)-\nabla \cdot \frac{1}{\mu_{t r}} \int_{(4 \pi)} \mathrm{d}^{2} \hat{\boldsymbol{s}} \hat{\boldsymbol{s}} \varepsilon_{r i}(\boldsymbol{r}, t)
$$

In our FDTD analysis, we employ Eqs. (1) and omit the source term first to discuss the evolution of the error with the time increase. The FDTD equations without the source term are expressed as below,

$$
\begin{aligned}
J_{X}^{n+\frac{1}{2}}\left(i+\frac{1}{2}, j, k\right) & =C_{x}^{J J} J_{X}^{n-\frac{1}{2}}\left(i+\frac{1}{2}, j, k\right)-C_{x}^{J \Phi}\left[\Phi^{n}(i+1, j, k)-\Phi^{n}(i, j, k)\right] \\
J_{Y}^{n+\frac{1}{2}}\left(i, j+\frac{1}{2}, k\right) & =C_{y}^{J J} J_{Y}^{n-\frac{1}{2}}\left(i, j+\frac{1}{2}, k\right)-C_{y}^{J \Phi}\left[\Phi^{n}(i, j+1, k)-\Phi^{n}(i, j, k)\right] \\
J_{Z}^{n+\frac{1}{2}}\left(i, j, k+\frac{1}{2}\right) & =C_{z}^{J J} J_{Z}^{n-\frac{1}{2}}\left(i, j, k+\frac{1}{2}\right)-C_{z}^{J \Phi}\left[\Phi^{n}(i, j, k+1)-\Phi^{n}(i, j, k)\right] \\
\Phi^{n+1}(i, j, k) & =C^{\Phi \Phi} \Phi^{n}(i, j, k)-C^{\Phi J}\left\{\frac{1}{\Delta x}\left[J_{X}^{n+\frac{1}{2}}\left(i+\frac{1}{2}, j, k\right)-J_{X}^{n+\frac{1}{2}}\left(i-\frac{1}{2}, j, k\right)\right]\right. \\
& +\frac{1}{\Delta y}\left[J_{Y}^{n+\frac{1}{2}}\left(i, j+\frac{1}{2}, k\right)-J_{Y}^{n+\frac{1}{2}}\left(i, j-\frac{1}{2}, k\right)\right] \\
& \left.+\frac{1}{\Delta z}\left[J_{Z}^{n+\frac{1}{2}}\left(i, j, k+\frac{1}{2}\right)-J_{Z}^{n+\frac{1}{2}}\left(i, j, k-\frac{1}{2}\right)\right]\right\}
\end{aligned}
$$


where

$$
\begin{aligned}
& C_{x}^{J J}=\frac{2-\mu_{t r}\left(i+\frac{1}{2}, j, k\right) v \Delta t}{2+\mu_{t r}\left(i+\frac{1}{2}, j, k\right) v \Delta t} \quad C_{y}^{J J}=\frac{2-\mu_{t r}\left(i, j+\frac{1}{2}, k\right) v \Delta t}{2+\mu_{t r}\left(i, j+\frac{1}{2}, k\right) v \Delta t} \quad C_{z}^{J J}=\frac{2-\mu_{t r}\left(i, j, k+\frac{1}{2}\right) v \Delta t}{2+\mu_{t r}\left(i, j, k+\frac{1}{2}\right) v \Delta t} \\
& C_{x}^{J \Phi}=\frac{2 v \Delta t}{3 \Delta x\left(2+\mu_{t r}\left(i+\frac{1}{2}, j, k\right) v \Delta t\right)} \quad C_{y}^{J \Phi}=\frac{2 v \Delta t}{3 \Delta y\left(2+\mu_{t r}\left(i, j+\frac{1}{2}, k\right) v \Delta t\right)} \quad C_{z}^{J \Phi}=\frac{2 v \Delta t}{3 \Delta z\left(2+\mu_{t r}\left(i, j, k+\frac{1}{2}\right) v \Delta t\right)} \\
& C^{\Phi \Phi}=\frac{2-v \Delta t \mu_{a}(i, j, k)}{2+v \Delta t \mu_{a}(i, j, k)} \quad C^{\Phi J}=\frac{2 v \Delta t}{2+v \Delta t \mu_{a}(i, j, k)}
\end{aligned}
$$

$\Delta x, \Delta y$ and $\Delta z$ are the size of the spatial discretization cell. $\Delta t$ is the time increment. The indices $i, j$ and $k$ denote the positions of the nodes in the mesh and the index $n$ denotes the time step.

\section{The von Neumann stability analysis}

The von Neumann stability analysis is a very useful tool for determining the stability condition of the difference equations $[12,13]$, and is based on the Fourier decomposition of numerical error. Take $U^{n}$ as a vector at step $\mathrm{n}$ in the finite difference scheme, while $\hat{U}_{\boldsymbol{q}}^{n}$ is the single Fourier component of the field vector $U^{n}$, where $\boldsymbol{q}$ is the spatial wave number. Let us consider a homogeneous equation $\hat{U}_{q}^{n+1}=G \hat{U}_{q}^{n}$ derived from a difference scheme, where the Matrix $G$ is called the amplification matrix. This equation expresses the evolution of the vector $U^{n}$. Inductively, we know $\hat{U}_{q}^{n}=G^{n} \hat{U}_{q}^{0}$. The time evolution of the round-off error is determined by the same homogeneous equation. We can estimate the error in step $n$ as

$$
\varepsilon_{n}=\varepsilon_{0} \alpha^{n}
$$

where $\alpha$ is the eigenvalue of the matrix $G$ and $\varepsilon_{0}$ is the initial error. This means when the eigenvalue is larger than one, the error grows exponentially with time evolution. In order to stabilize the scheme, all of the eigenvalues of the amplification matrix should be less than one.

3.1 Applying the von Neumann stability analysis to the FDTD solution of the optical diffusion equations

Now we assume $\mu_{a}$ and $\mu_{t r}$ are constant and there are no sources. Considering a single harmonics expressed by spatial wave number $\boldsymbol{q}=\left(q_{x}, q_{y}, q_{z}\right)$, we can write the fluence rate and radiant flux as

$$
\begin{aligned}
J_{X}^{n+\frac{1}{2}}\left(i+\frac{1}{2}, j, k\right) & =J_{X}^{n+\frac{1}{2}} \mathrm{e}^{\mathrm{i}\left(q_{x} \Delta x\left(i+\frac{1}{2}\right)+q_{y} \Delta y j+q_{z} \Delta z k\right)} \\
J_{Y}^{n+\frac{1}{2}}\left(i, j+\frac{1}{2}, k\right) & =J_{Y}^{n+\frac{1}{2}} \mathrm{e}^{\mathrm{i}\left(q_{x} \Delta x i+q_{y} \Delta y\left(j+\frac{1}{2}\right)+q_{z} \Delta z k\right)} \\
J_{Z}^{n+\frac{1}{2}}\left(i, j, k+\frac{1}{2}\right) & =J_{Z}^{n+\frac{1}{2}} \mathrm{e}^{\mathrm{i}\left(q_{x} \Delta x i+q_{y} \Delta y j+q_{z} \Delta z\left(k+\frac{1}{2}\right)\right)} \\
\Phi^{n}(i, j, k) & =\Phi^{n} \mathrm{e}^{\mathrm{i}\left(q_{x} \Delta x i+q_{y} \Delta y j+q_{z} \Delta z k\right)}
\end{aligned}
$$


where $J_{X}^{n+1 / 2}, J_{Y}^{n+1 / 2}, J_{Z}^{n+1 / 2}$ and $\Phi^{n}$ are coefficients, which depend only on the time step $n$. Substituting these equations above into Eqs. (2), we get the homogeneous equation,

$$
\varphi_{n+1}=G \varphi_{n}
$$

where



$$
\begin{aligned}
& \varphi_{n}=\left(\begin{array}{c}
\Phi^{n} \\
J_{X}^{n-\frac{1}{2}} \\
J_{Y}^{n-\frac{1}{2}} \\
J_{Z}^{n-\frac{1}{2}}
\end{array}\right)
\end{aligned}
$$

and

$$
B=\frac{16 \Delta t^{2} v^{2}}{\left(2+v \Delta t \mu_{a}\right)\left(2+v \Delta t \mu_{t r}\right)} P
$$

where

$$
P=\frac{1}{3}\left(\frac{\sin ^{2}\left(\frac{q_{x} \Delta x}{2}\right)}{\Delta x^{2}}+\frac{\sin ^{2}\left(\frac{q_{y} \Delta y}{2}\right)}{\Delta y^{2}}+\frac{\sin ^{2}\left(\frac{q_{z} \Delta z}{2}\right)}{\Delta z^{2}}\right)
$$

We want to know the condition that all the eigenvalues of the amplification matrix $G$ are less than one. After some calculations, we can get three eigenvalues of the matrix,

$$
\begin{gathered}
\gamma_{J J}=C^{J J} \\
\gamma_{ \pm}=\frac{C^{\Phi \Phi}+C^{J J}-B \pm \sqrt{\left(B+C^{J J}-C^{\Phi \Phi}\right)^{2}-4 B C^{J J}}}{2}
\end{gathered}
$$

The eigenvalue $\gamma_{J J}$ is degenerate. Let us consider the condition that the absolute values of the three eigenvalues are not larger than one. Since $\mu_{t r}, \mu_{a}, \Delta x, \Delta y, \Delta z$, $\Delta t, \varepsilon$ and $P$ are all positive, we can deduce the relation $\left|\gamma_{J J}\right|<1$, and we prove in Appendix A that

$$
\begin{array}{ll}
\left|\gamma_{ \pm}\right|<1 \text { as } & v \Delta t<1 / \sqrt{P} \\
\left|\gamma_{-}\right| \geq 1 \text { as } & v \Delta t \geq 1 / \sqrt{P}
\end{array}
$$


From the relations (10) and (11), the condition under which all the eigenvalues are less than one is

$$
v \Delta t<\frac{1}{\sqrt{P}}
$$

$P$ depends on the spatial wave number components $q_{x}, q_{y}$ and $q_{z}$, and these values have upper and lower limits because of the Nyquist theorem. For example $q_{x}$ can take values between $-\pi / \Delta x$ and $\pi / \Delta x$. For any spatial wave number, the inequality (12) must be satisfied, and therefore

$$
v \Delta t<\sqrt{\frac{3}{\Delta x^{-2}+\Delta y^{-2}+\Delta z^{-2}}}(3-\mathrm{D})
$$

This is the stability condition for FDTD of diffusion equations for the three dimensional case (3-D) and is our core result of this paper. The stability condition in the one dimensional case (1-D) can be derived by substituting $q_{y}=q_{z}=0$ into Eq. (7) because the field values $J_{x}, J_{y}, J_{z}$ and $\Phi$ are independent of $y$ and $z$ in Eqs. (2). In this case, $P$ is the value from 0 to $\Delta x^{-2} / 3$, then the stability condition becomes

$$
v \Delta t<\sqrt{3} \Delta x \quad(1-\mathrm{D})
$$

In the same way, the stability condition in the two dimensional case (2-D) is

$$
v \Delta t<\sqrt{\frac{3}{\Delta x^{-2}+\Delta y^{-2}}}(2-\mathrm{D})
$$

Let us note that the factors in Eqs. (13), (14) and (15) originate from the factor $1 / 3$ in the second term on the left hand side of the first equation of Eqs. (1). The left hand side of the diffusion equation for 2-D corresponding to the first equation of Eqs. (1) is the form of

$$
\frac{1}{v} \frac{\partial \boldsymbol{J}(\boldsymbol{r}, t)}{\partial t}+\frac{1}{2} \nabla \phi(\boldsymbol{r}, t)+\mu_{t r} \boldsymbol{J}(\boldsymbol{r}, t)
$$

And the preconstant of $\nabla \phi(\boldsymbol{r}, t)$ becomes $1 / 2$ instead of 1/3. In Eqs. (14) and (15), the stability conditions for 1-D and 2-D are derived from Eqs. (1), not from (16). Therefore "2-D" is the condition where all the values such as $\mu_{t r}$ are constant along the $\mathrm{z}$-axis in the 3-D region, and "1-D" is the condition where all the values are constant along the $\mathrm{y}$-axis and $\mathrm{z}$-axis in the $3 \mathrm{D}$ region. When $\Delta x=\Delta y=\Delta z$ and denoting the dimension of the field by $d$, the stability condition becomes

$$
\lambda=v \Delta t / \Delta x<\sqrt{3 / d}(d=1,2,3)
$$

$\lambda$ is usually called the Courant number or the Courant factor [11]. 


\section{Numerical calculation}

In this section, we numerically verify the stability condition derived in previous sections. First, we calculate the time development of the photon fluence rate $\Phi$ for some initial conditions. In order to see the discrepancy near the stability bound, we choose two Courant numbers, one of which is $1 \%$ larger and the other is $1 \%$ smaller than the stability bound in different dimension. Second, we demonstrate the stability condition for a pulsed source incident on a diffuse material similar to biological tissue.

\subsection{Results around stability bound}

We assume a homogeneous media with a refractive index $n_{m}=1.5$, an absorption coefficient $\mu_{a}=0.001 \mathrm{~mm}^{-1}$, and a reduced scattering coefficient $\mu_{s}^{\prime}=1 \mathrm{~mm}^{-1}$. A space divided into $100 \times 100 \times 10$ cubic cells with $\Delta x=\Delta y=\Delta z=0.5 \mathrm{~mm}$ is used and the periodic boundary condition is applied.

To see the dimensional dependence, the simulation starts with the initial distribution of the photon fluence rate at $t=0$ or $n=0$,

$$
\Phi_{0}^{3 D}(x, y, z)=1-\frac{1}{3} \cos \left(\frac{2 \pi x}{p_{x}}\right)-\frac{1}{3} \cos \left(\frac{2 \pi y}{p_{y}}\right)-\frac{1}{3} \cos \left(\frac{2 \pi z}{p_{z}}\right)
$$

where $p_{x}, p_{y}$ and $p_{z}$ are the periods of the initial distribution along each axis and we put $p_{x}=p_{y}=p_{z}=20 \Delta x$. In this condition the stability bound is $\lambda=1$ from Eq. (17) because $d=3$.

Fig. 1 presents the results of the simulation. To see the error growth rate, we plot the time dependence of the peak-to-valley $(\mathrm{P}-\mathrm{V})$ of the photon fluence rate over the entire region. The theoretical curve calculated from Eq. (4) (see Appendix C) is plotted as a dotted line. Under the stability bound, where the Courant number is $\lambda=0.99$, the numerical results match the theoretical result. On the other hand, when the Courant number is $1 \%$ above the stability bound, the eigenvalue is $\gamma_{-} \cong-1.135$, indicating an unstable condition. The P-V of the photon fluence rate $\Phi$ grows exponentially from around 200 time steps. We can estimate the error by using Eq. (4). At the beginning, the round-off error is about $10^{-15}$, and this value is determined by the number of significant figures of the data type used in the numerical calculation. Here we used the double-precision floating point number in a Java program (JDK1.7.0_01 on Redhat Enterprise Linux 5 64bit) and the number of significant figures was approximately 15 to 17 decimal digits. The growth in the simulation is very close to the theoretical prediction.

Fig. 1(b) is an example of the $\Phi$ distribution of the X-Y slice, which is depicted by square pixels, at the 190th time-step under the unstable condition in which the Courant factor $\lambda=1.01$. We can see that the unstable field pattern has the form of a checkerboard. This checkerboard pattern originates from the round-off error and grows exponentially according to Eq. (4). Fig. 1(c) shows the cross-sectional $\Phi$ values of Fig. 1(b) along the x-direction at $y=25 \mathrm{~mm}$. The period of the error is 2-pixels because of the checkerboard pattern. The absolute value of eigenvalue $\gamma_{-}$ is a monotonically increasing function of $\mathrm{P}$ and $\mathrm{P}$ takes the maximum value when 

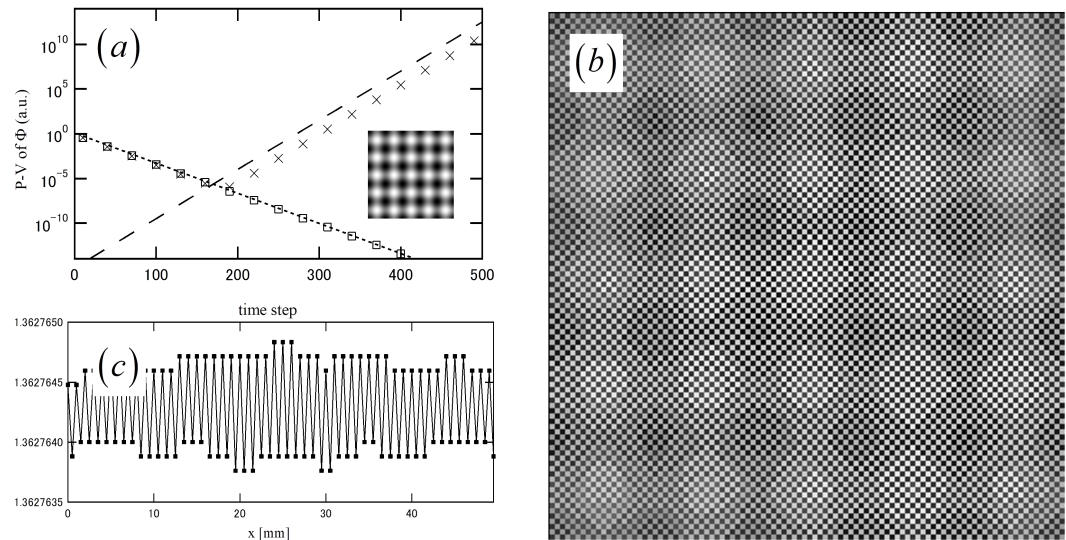

Fig. 1 Simulation results around the stability bound. 3D initial distribution is expressed by Eq. (18). (a) The peak-to-valley (P-V) of $\Phi$ in each time step. $\square: \lambda=0.99, \times: \lambda=1.01$, and dashed line is $C\left|\gamma_{-}\right|^{n}$. Dotted line is the theoretical line of the amplitude. Inside the graph, initial X-Y distribution of $\Phi^{0}$ is expressed by Eq. (18). (b) $\Phi$ distribution of X-Y slice at 190th time-step under the unstable condition in which the Courant factor $\lambda=1.01$. (c) The cross-sectional $\Phi$ values of the Fig. 1(b).

Table 1 Numerical condition and expected stability. Each initial distribution is expressed by Eqs. (30a), (30b) and (18).

\begin{tabular}{lclr}
\hline & Initial distribution & $\lambda$ & Stability \\
\hline 1 & $\Phi_{0}^{1 D}(x, y, z)$ & $0.99 \sqrt{3}$ & Stable \\
2 & $\Phi_{0}^{1 D}(x, y, z)$ & $1.01 \sqrt{3}$ & Unstable \\
3 & $\Phi_{0}^{2 D}(x, y, z)$ & $0.99 \sqrt{3 / 2}$ & Stable \\
4 & $\Phi_{0}^{2 D}(x, y, z)$ & $0.99 \sqrt{3 / 2}$ & Unstable \\
5 & $\Phi_{0}^{3 D}(x, y, z)$ & 0.99 & Stable \\
6 & $\Phi_{0}^{3 D}(x, y, z)$ & 1.01 & Unstable \\
\hline
\end{tabular}

$q_{x}=q_{y}=q_{z}=\pi / \Delta x$, under which error forms the checkerboard pattern. Hence the checkerboard pattern error increases most rapidly.

We have shown the results for a 3D distribution here. We also show the results for the other dimensions, 1D and 2D in Appendix B. All the results indicate that the stability condition follows Eq. (17), and clearly depends on the dimension of the distribution as we have indicated. Table 1 summarizes the above results.

\subsection{A pulse light incident on a cubic diffuse material}

We also show results of a more realistic simulation; a pulse light is injected at a point on the surface of a cubic diffuse material. We apply the Robin boundary condition [7]. This boundary condition assumes $\Phi=0$ on the extrapolated boundary, which is $2 A / 3 \mu_{t r}$ apart from the real boundary. The coefficient $A$ depends on the relative refractive index between the medium and the outside space. We assume that the incident beam is a Gaussian pulse with duration of about $40 \Delta t$. We apply the point source 

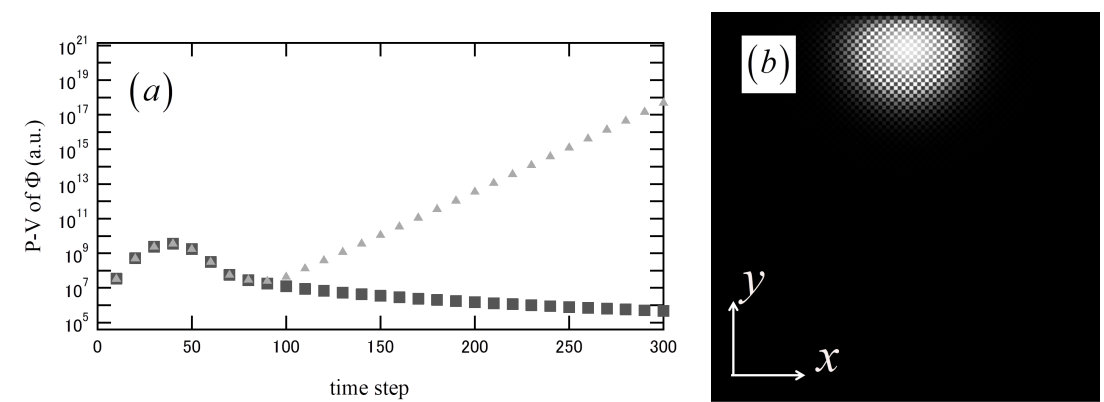

Fig. 2 Simulation of the point source approximation with Robin boundary condition. (a) peak-to-valley (P-V) of distribution $\Phi^{0}$ in each time step. $\lambda=0.99$ (square dot) and $\lambda=1.01$ (triangle dot). (b) A x-y distribution of photon fluence rate $\Phi$ at 90 -time step with $\lambda=1.01$.

approximation [7], where the incident source is a point source. The point source is placed at the center and $1 \mathrm{~mm}$ inside from the real boundary, or $3 \mathrm{~mm}$ inside from the extrapolated boundary of the $x-y$ plane surface of the cubic material.

Again, the grid size is $100 \times 100 \times 100$ and the spatial sampling pitch is $\Delta x=$ $\Delta y=\Delta z=0.5 \mathrm{~mm}$. Therefore we assume that the light pulse is incident on the center of the surface of the $50 \mathrm{~mm} \times 50 \mathrm{~mm} \times 50 \mathrm{~mm}$ cubic diffuse material. The refractive index of the material is set to be $n_{m}=1.4$, while the absorption and the reduced scattering coefficients are $\mu_{a}=0.005 \mathrm{~mm}^{-1}$ and $\mu_{s}^{\prime}=1 \mathrm{~mm}^{-1}$, respectively.

As in the previous simulation, we use $\gamma=0.99$ which is $1 \%$ smaller than the stability bound, and $\gamma=1.01$ which is $1 \%$ larger than the stability bound. Fig. 2 shows the results of this simulation. Fig. 2(a) shows the P-V of the photon fluence in the simulation region. These results also support that the stability bound expressed by Eq. (17). Fig. 2(b) shows the photon fluence rate $\Phi$ on the X-Y plane at the 90 th timestep with $\gamma=1.01$, which is an unstable condition. We can also see the checkerboard pattern that is characteristic of the growing round-off error in this result.

\section{Conclusion}

We derived the stability condition of the FDTD method for the optical diffusion equations analytically in each dimension using the von Neumann stability analysis, and then numerically demonstrated the validity of the stability bound. As the results show, our formulation enables a stable application of the FDTD method for the optical diffusion equations.

\section{Acknowledgment}

We wish to thank Dr. Daniel G. Smith and Setsuko Iwami of Nikon Research Corporation of America (NRCA) and Yuichi Takigawa for their great help. 
Appendix A. Proof of inequalities (10) and (11)

In this appendix, we prove the inequalities (10) and (11). We rewrite these inequalities below

$$
\begin{array}{ll}
\left|\gamma_{ \pm}\right|<1 \text { as } & v \Delta t<1 / \sqrt{P} \\
\left|\gamma_{-}\right| \geq 1 \text { as } & v \Delta t \geq 1 / \sqrt{P}
\end{array}
$$

We don't need to know the value $\left|\gamma_{+}\right|$as $v \Delta t>1 / \sqrt{P}$. Whether the value $\left|\gamma_{+}\right|$is less than one or not, this FDTD is unstable when $v \Delta t>1 / \sqrt{P}$, because the other eigenvalue $\left|\gamma_{-}\right|$is larger than one as we prove below. Substituting Eqs. (3) and (6) into Eq. (9),

$$
\gamma_{ \pm}=g_{1} \pm g_{2}
$$

where

$$
\begin{aligned}
g_{1} & =\frac{4-\left(\mu_{a} \mu_{t r}+8 P\right) v^{2} \Delta t^{2}}{\left(2+v \Delta t \mu_{a}\right)\left(2+v \Delta t \mu_{t r}\right)} \\
g_{2} & =\frac{2 v \Delta t \sqrt{4 P\left(\mu_{a} \mu_{t r}+4 P\right) v^{2} \Delta t^{2}-16 P+\left(\mu_{t r}-\mu_{a}\right)^{2}}}{\left(2+v \Delta t \mu_{a}\right)\left(2+v \Delta t \mu_{t r}\right)}
\end{aligned}
$$

Now substituting $v \Delta t=1 / \sqrt{P}$ into Eq. (19), we get

$$
\begin{aligned}
& \left.\gamma_{+}\right|_{v \Delta t=1 / \sqrt{P}}=-\frac{\left(2-P^{-1 / 2} \mu_{a}\right)\left(2-P^{-1 / 2} \mu_{t r}\right)}{\left(2+P^{-1 / 2} \mu_{a}\right)\left(2+P^{-1 / 2} \mu_{t r}\right)} \\
& \left.\gamma_{-}\right|_{v \Delta t=1 / \sqrt{P}}=-1
\end{aligned}
$$

Around $v \Delta t=1 / \sqrt{P}, \gamma_{-}$can be larger than one, hence $v \Delta t=1 / \sqrt{P}$ is the candidate for stability bound. Considering the function $\left|\frac{2-x}{2+x}\right|$ as $x>0$, we get $\left|\gamma_{+}\right|_{v \Delta t=1 / \sqrt{P}} \mid<$

The square root in $g_{2}$ is zero if

$$
v \Delta t=\sqrt{\frac{16 P-\left(\mu_{t r}-\mu_{a}\right)^{2}}{4 P\left(\mu_{a} \mu_{t r}+4 P\right)}}
$$

Then we define $\beta_{r}$ by,

$$
\beta_{r}{ }^{2}=\frac{16 P-\left(\mu_{t r}-\mu_{a}\right)^{2}}{4 P\left(\mu_{a} \mu_{t r}+4 P\right)}
$$

Since we know that $\left(\mu_{t r}-\mu_{a}\right)^{2}>0$ and $\mu_{a}, \mu_{t r}>0$

$$
\beta_{r}{ }^{2}=\frac{16 P-\left(\mu_{t r}-\mu_{a}\right)^{2}}{4 P\left(\mu_{a} \mu_{t r}+4 P\right)}<\frac{16}{4^{2} P}=\left(\frac{1}{\sqrt{P}}\right)^{2}
$$

Therefore, the region over which $g_{2}$ is not real is smaller than $v \Delta t=1 / \sqrt{P}$. Fig. 3 depicts this relation. Note that if $16 P \leq\left(\mu_{t r}-\mu_{a}\right)^{2}$, then $g_{2}$ is real for any $v \Delta t>0$.

If we can prove the states (A1) and (A2) below, we show that $\left|\gamma_{ \pm}\right| \leq 1$ as $v \Delta t \leq$ $1 / \sqrt{P}$, and (A3) leads $\left|\gamma_{ \pm}\right|>1$ as $v \Delta t>1 / \sqrt{P}$. We can therefore prove what we set out to prove in this appendix. 
Fig. 3 The schematic behavior of $\gamma_{ \pm}$.

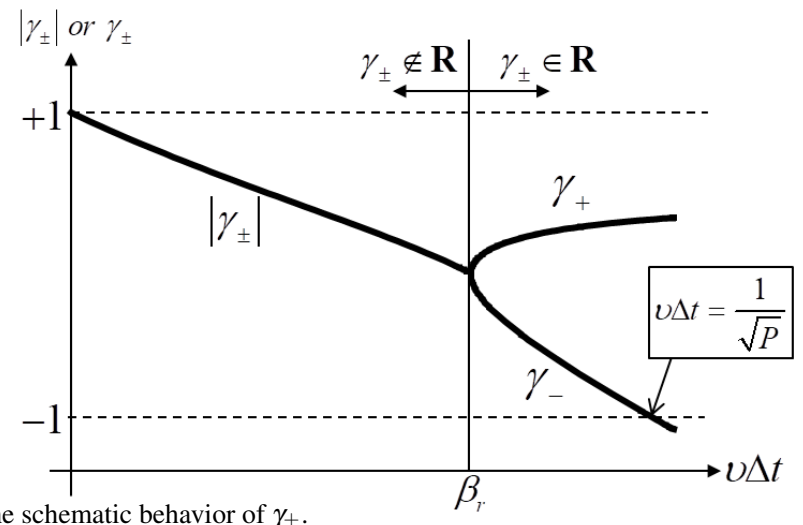

(A1) $\left|\gamma_{ \pm}\right|<1$ as $0<v \Delta t \leq \beta_{r}$

(A2) $\left|\gamma_{+}\right|<1,\left|\gamma_{-}\right|<1$ as $v \Delta t \in\left(\beta_{r}, 1 / \sqrt{P}\right)$

(A3) $\gamma_{-}=-1$ as $v \Delta t=1 / \sqrt{P}$, and $\gamma_{-}$monotonically decreases as $v \Delta t>\beta_{r}$

Proof of (A1) We can obtain $\left|\gamma_{ \pm}\right|^{2}$ from Eq. (19)

$$
\left|\gamma_{ \pm}\right|^{2}=g_{1}^{2}+g_{2}^{2}=\frac{\left(2-v \Delta t \mu_{a}\right)^{2}\left(2-v \Delta t \mu_{t r}\right)^{2}}{\left(2+v \Delta t \mu_{a}\right)^{2}\left(2+v \Delta t \mu_{t r}\right)^{2}}
$$

function $\left|\frac{2-x}{2+x}\right|$ is less than unity as $x>0$, therefore, as we have considered in Eq. (21a), we get $\left|\gamma_{ \pm}\right|<1$.

Proof of (A2) As for $\gamma_{+}=g_{1}+g_{2},\left|\gamma_{+}\right|=\left|g_{1}+g_{2}\right| \leq+1$ is equivalent to two inequalities $-1-g_{1} \leq g_{2}$ and $g_{2} \leq 1-g_{1}$. First we show $-1-g_{1} \leq g_{2}$. Consider the region $v \Delta t<1 / \sqrt{P}$, so that $1-v^{2} \Delta t^{2} P>0$, and

$$
-1-g_{1}=-\frac{8\left(1-P v^{2} \Delta t^{2}\right)+2 v \Delta t\left(\mu_{a}+\mu_{t r}\right)}{\left(2+v \Delta t \mu_{a}\right)\left(2+v \Delta t \mu_{t r}\right)}<0
$$

Because apparently $g_{2}>0$, so that $-1-g_{1} \leq g_{2}$. Next, we want to show that $-1-$ $g_{1} \leq g_{2}$, and so we can calculate

$$
\left(1-g_{1}\right)^{2}-g_{2}^{2}=\frac{4 v^{2} \Delta t^{2}\left(4 P+\mu_{a} \mu_{t r}\right)}{\left(2+v \Delta t \mu_{a}\right)\left(2+v \Delta t \mu_{t r}\right)}>0
$$

Therefore $g_{2}^{2} \leq\left(1-g_{1}\right)^{2} \cdot g_{2}$ is positive and

$$
1-g_{1}=\frac{2 v \Delta t\left(4 P v \Delta t+\mu_{t r}+\mu_{a}+v \Delta t \mu_{a} \mu_{t r}\right)}{\left(2+v \Delta t \mu_{a}\right)\left(2+v \Delta t \mu_{t r}\right)}>0
$$

because all the parameters in this equation are positive. Since the square function is an increasing monotonic function in the positive domain, we have $g_{2} \leq 1-g_{1}$. We have shown that $\left|\gamma_{+}\right| \leq 1$ as $v \Delta t \in\left(\beta_{r}, 1 / \sqrt{P}\right)$. Although it is omitted here, the bound on $\gamma_{-}$can be shown in the same way. 
Proof of (A3) We have shown that $\gamma_{-}=-1$ as $v \Delta t=1 / \sqrt{P}$ in Eq.(21b), so it only remains to show that $\gamma_{-}$monotonically decreases as for $v \Delta t$.

Differentiating $g_{2}{ }^{2}$ with respect to $v \Delta t>0$,

$$
\begin{aligned}
\frac{\partial g_{2}^{2}}{\partial(v \Delta t)}= & \frac{32 P v \Delta t\left(4 P+\mu_{a} \mu_{t r}\right)}{\left(2+v \Delta t \mu_{a}\right)^{3}\left(2+v \Delta t \mu_{t r}\right)^{3}}\left(4\left(v^{2} \Delta t^{2}-\beta_{r}{ }^{2}\right)+4 v^{2} \Delta t^{2}+2 v^{2} \Delta t^{3}\left(\mu_{t r}+\mu_{a}\right)+v^{2} \Delta t^{2} \beta_{r}{ }^{2} \mu_{t r} \mu_{a}\right) \\
& >0
\end{aligned}
$$

Therefore $g_{2}^{2}$ is monotonically increasing. Because $g_{2}>0, g_{2}$ is also monotonically increasing.

Differentiating $g_{1}$,

$\frac{\partial g_{1}}{\partial(v \Delta t)}=-\frac{\left(v^{2} \Delta t^{2} \mu_{a} \mu_{t r}+4+8 P v^{2} \Delta t^{2}\right)\left(\mu_{a}+\mu_{t r}\right)}{\left(2+v \Delta t \mu_{a}\right)^{2}\left(2+v \Delta t \mu_{t r}\right)^{2} / 2}-\frac{8 v \Delta t \mu_{t r} \mu_{a}+32 P v \Delta t}{\left(2+v \Delta t \mu_{a}\right)^{2}\left(2+v \Delta t \mu_{t r}\right)^{2} / 2}<0$

shows that $g_{1}$ is monotonically decreasing and $\gamma_{-}=g_{1}-g_{2}$ is also monotonically decreasing.

\section{Appendix B. The other results of the simulation of the different initial distribu-} tion

As shown in section 3 , the stability condition depends on the dimensions of the field; $\lambda=\sqrt{3}$ in $1 \mathrm{D}$, and $\lambda=\sqrt{3 / 2}$ in $2 \mathrm{D}$. We therefore include the results of the simulation in these dimensions. We use the same simulation parameters as in section 4 except that the initial conditions, as stated in Eq. (18) for the 3-D case, become Eqs. (30a) and (30b) for 1-D and 2-D, respectively.

$$
\begin{aligned}
& \Phi_{0}^{1 D}(x, y, z)=1-\cos \left(\frac{2 \pi x}{p_{x}}\right) \\
& \Phi_{0}^{2 D}(x, y, z)=1-\frac{1}{2} \cos \left(\frac{2 \pi x}{p_{x}}\right)-\frac{1}{2} \cos \left(\frac{2 \pi y}{p_{y}}\right)
\end{aligned}
$$

where $p_{x}$ and $p_{y}$ are the period of the initial distribution along each axis and we put $p_{x}=p_{y}=20 \Delta x$. As in the 3D simulation, we choose the Courant numbers to be $1 \%$ larger and $1 \%$ lower than the stability bound. Fig. 4 shows that the results again support the boundary condition expressed by Eq. (17).

\section{Appendix C. The theoretical curve in the single mode distribution in the first numerical simulation}

In this appendix, we calculate the time dependence of the peak-to-valley when the initial distribution is expressed by Eq. (18).

Because the diffusion equations are linear, we only need to consider these terms. 

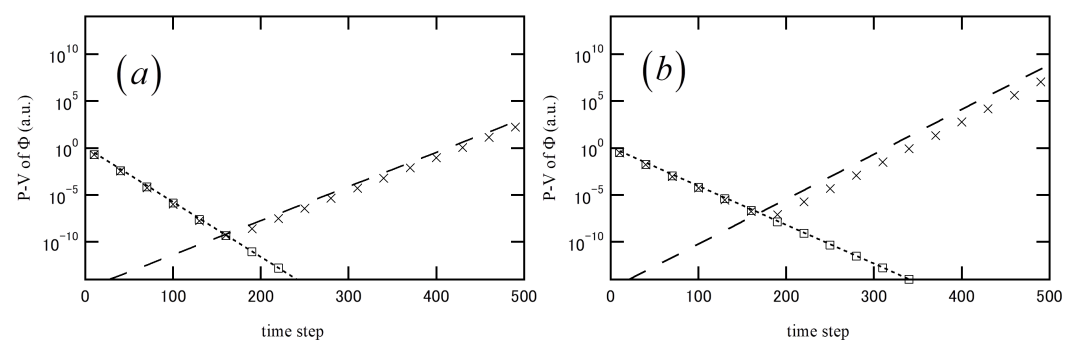

Fig. 4 Simulation results around stability bound in 1D and 2D. The peak-to-valley (P-V) of $\Phi$ in each time step (a) with the 1D initial distribution expressed by Eq. (30a) and (b) with the 2D initial distribution expressed by Eq. (30b). $\square: \lambda=0.99 \sqrt{3 / d}, \times: \lambda=1.01 \sqrt{3 / d}$, and dashed line is $C\left|\gamma_{-}\right|^{n} . \gamma_{-} \cong-1.0872$ when $\lambda=1.01 \sqrt{3}$ in $1 \mathrm{D}$-simulation, and $\gamma_{-} \cong-1.1162$ when $\lambda=1.01 \sqrt{3 / 2}$ in $2 \mathrm{D}$-simulation. The dotted line indicates the theoretical amplitude.

$$
\begin{aligned}
\phi(x, y, z ; t) & =b_{\phi} \exp \left(\frac{2 \pi \mathrm{i} x}{p_{x}}\right) \\
\boldsymbol{J}(x, y, z ; t) & =\left(\begin{array}{c}
b_{j} \exp \left(\frac{2 \pi \mathrm{i} x}{p_{x}}\right) \\
0 \\
0
\end{array}\right)
\end{aligned}
$$

where $b_{\phi}$ and $b_{j}$ depend only on time. $p_{x}$ is a spatial frequency.

Substitute the above equations into

$$
\begin{aligned}
& \frac{1}{v} \frac{\partial \boldsymbol{J}(\boldsymbol{r}, t)}{\partial t}+\frac{1}{3} \nabla \phi(\boldsymbol{r}, t)+\mu_{t r} \boldsymbol{J}(\boldsymbol{r}, t)=0 \\
& \frac{1}{v} \frac{\partial \phi(\boldsymbol{r}, t)}{\partial t}+\nabla \cdot \boldsymbol{J}(\boldsymbol{r}, t)+\mu_{a} \phi(\boldsymbol{r}, t)=0
\end{aligned}
$$

We then obtain,

$$
\frac{\partial}{\partial t}\left(\begin{array}{c}
b_{\phi} \\
b_{j}
\end{array}\right)=-v\left(\begin{array}{cc}
\mu_{a} & \frac{2 \pi \mathrm{i}}{p_{x}} \\
\frac{2 \pi \mathrm{i}}{3 p_{x}} & \mu_{t r}
\end{array}\right)\left(\begin{array}{c}
b_{\phi} \\
b_{j}
\end{array}\right)
$$

This differential equation is solved by orthogonalizing the matrix in this equation,

$$
\frac{\partial}{\partial t}\left(\begin{array}{l}
b_{\phi} \\
b_{j}
\end{array}\right)=-v P^{-1} \Lambda P\left(\begin{array}{c}
b_{\phi} \\
b_{j}
\end{array}\right)
$$

where

$$
\begin{gathered}
\Lambda=\left(\begin{array}{cc}
\lambda_{-} & 0 \\
0 & \lambda_{+}
\end{array}\right) \\
P=\left(\begin{array}{c}
p_{+} \\
p_{-}
\end{array}\right) \\
\lambda_{ \pm}=\frac{1}{2}\left(\mu_{a}+\mu_{t r}\right) \pm \frac{\sqrt{9 p_{x}^{2}\left(\mu_{a}-\mu_{t r}\right)^{2}-48 \pi^{2}}}{6 p_{x}} \\
p_{ \pm}=\frac{i}{4 \pi}\left[3 p_{x}\left(\mu_{t r}-\mu_{a}\right) \pm \sqrt{9 p_{x}^{2}\left(\mu_{a}-\mu_{t r}\right)^{2}-48 \pi^{2}}\right]
\end{gathered}
$$


We then obtain the solution with the initial values $b_{\phi}=1$ and $b_{j}=b_{j}^{0}$ at $t=0$,

$$
\left(\begin{array}{c}
b_{\phi} \\
b_{j}
\end{array}\right)=P^{-1} \exp (-v \Lambda t) P\left(\begin{array}{c}
b_{\phi}^{0} \\
b_{j}^{0}
\end{array}\right)
$$

In the simulation described in the article, we can use $b_{j}^{0}$ and from the $(1,1)$-element of $P^{-1} \exp (-v \Lambda t) P$ we have the solution of the differential equation,

$$
\begin{aligned}
\phi(x, y, z ; t) & =b_{\phi}{ }^{0} \exp \left(\frac{2 \pi \mathrm{i} x}{p_{x}}\right) \exp \left[-v t\left(\frac{\mu_{a}+\mu_{t r}}{2}+\frac{\eta}{6 p_{x}}\right)\right] \\
& \times\left\{\left(\frac{1}{2}-\frac{3 p_{x}\left(\mu_{a}-\mu_{t r}\right)}{2 \eta}\right) \exp \left(\frac{v \eta}{3 p_{x}} t\right)+\frac{1}{2}+\frac{3 p_{x}\left(\mu_{a}-\mu_{t r}\right)}{2 \eta}\right\}
\end{aligned}
$$

where $\eta=\sqrt{9 p_{x}^{2}\left(\mu_{a}-\mu_{t r}\right)^{2}-48 \pi^{2}}$

Finally, we obtain the theoretical time dependence of peak-to-valley as,

$2 b_{\phi}{ }^{0} \exp \left[-v t\left(\frac{\mu_{a}+\mu_{t r}}{2}+\frac{\eta}{6 p_{x}}\right)\right]\left\{\left(\frac{1}{2}-\frac{3 p_{x}\left(\mu_{a}-\mu_{t r}\right)}{2 \eta}\right) \exp \left(\frac{v \eta}{3 p_{x}} t\right)+\frac{1}{2}+\frac{3 p_{x}\left(\mu_{a}-\mu_{t r}\right)}{2 \eta}\right\}$

When $p_{x}>0$, this value is exponentially increasing as $t$ increases, and this expression shows that the increasing rate becomes higher as the spatial period $p_{x}$ becomes smaller. This is the reason why the error forms the checkerboard pattern whose spatial frequency is the highest in the 2D image, as shown in Fig. 1(b) or Fig. 2.

\section{References}

1. M. L. Adams and E. W. Larsen, "Fast iterative methods for discrete ordinates particle transport calculations, ” Prog. Nucl. Energy,40, 3(2002)

2. J. K. Fletcher, "A solution of the neutron transport equation using spherical harmonics, ” J. Phys. A: Math. Gen. 16,2827. (1983)

3. K. Kobayashi, H. Oigawa, and H. Yamagata, " The spherical harmonics method for the multigroup transport equation in X-y geometry, ” Ann. Nucl. Energy 13, 663 (1986)

4. A. D. Klose and E. W. Larsen "Light transport in biological tissue based on the simplified spherical harmonics equations." J Comput Phys. 220.441.(2006)

5. A. K. Jha , M. A. Kupinski , T. Masumura , E. Clarkson , A. A. Maslov , H. H. Barrett, “ Simulating photon-transport in uniform media using the radiative transfer equation: A study using the Neumannseries approach,” J. Opt. Soc. Amer. A. 29, 1741 (2012)

6. S. R. Arridge, "Optical tomography in medical imaging," Inverse Problem, 15, R41-R93(1999)

7. F. Martelli, S. D. Bianco, A. Ismaelli and G. Zaccanti, "Light Propagation through Biological Tissue and Other Diffusive Media," SPIE press (2010)

8. S. R. Arridge, M. Schweiger, M. Hiraoka and D. T. Delpy, “ A finite element approach for modeling photon transport in tissue, " Med. Phys. 20, 299(1993)

9. A. H. Hielscher, A. D. Klose and K. M. Hanson, "Gradient-Based Iterative Image Reconstruction Scheme for Time-Resolved Optical Tomography ” IEEE Trans. Medical Imaging. 18, 262 (1999)

10. T. Tanifuji and M. Hijikata, "Finite Difference Time Domain (FDTD) Analysis of Optical Pulse Responses in Biological Tissues for Spectroscopic Diffused Optical Tomography” IEEE Trans. Medical Imaging. 21, 181 (2002).

11. A. Taflove and and S. C. Hagness, "Computational electrodynamics The finite-difference time-domain method" 3rd ed. Artech House (2005)

12. C. Hirsch, "Numerical Computation of Internal and External Flows, Fundamentals of Numerical Discretization”, Wiley,Volume 1 (1989)

13. R. D. Richtmyer and K.W. Morton, “Difference Methods for Initial-Value Problems " 2nd ed,WileyInterscience (1967) 